\title{
The effects of vitamin D supplementation in carpal tunnel syndrome treatment outcomes: a systematic review
}

\author{
Chirathit Anusitviwat, Porames Suwanno and Sitthiphong Suwannaphisit *i)
}

\begin{abstract}
Purpose: Vitamin D deficiency is related to carpal tunnel syndrome symptoms. Correcting vitamin D levels by supplementation was supposed to improve carpel tunnel symptoms, though there is a lack of aggregated data about treatment outcomes. This study aimed to examine whether vitamin D supplementation could improve the treatment outcomes in carpal tunnel syndrome patients.

Methods: A comprehensive search of the PubMed, Cochrane Library, Scopus, and Web of Science databases for articles on vitamin D and carpel tunnel syndrome from January 2000 to March 2021 was performed. The article screening and data extraction were performed by two investigators independently with blinding to decisions on selected studies. All included studies had assessed the quality of evidence using the Methodological Index for Non-Randomized Studies (MINORS) scoring system.
\end{abstract}

Results: We retrieved four studies that met the eligibility criteria. The treatment outcomes were evaluated by visual analog scale (124 wrists), functional scores (176 patients), muscle strength (84 patients), and nerve conduction velocity (216 wrists). After vitamin D supplementation, two studies reported improved pain scores and nerve conduction velocity, and three studies showed enhancement of functional status.

Conclusion: Vitamin D administration could offer favorable outcomes in pain improvement, better functional status, and increased sensory conduction velocity in carpal tunnel syndrome. However, there is to date no recommendations concerning a standardized dose or duration of vitamin D administration in carpal tunnel syndrome; prescribing vitamin $\mathrm{D}$ at the usual appropriate dose is suggested as an additional treatment in patients with mild to moderate carpel tunnel symptoms.

Level of Evidence: Level IV, therapeutic study

Keywords: Carpal Tunnel Syndrome, Outcome, Vitamin D, Supplementation

\section{Introduction}

Carpal tunnel syndrome (CTS), peripheral compressive neuropathy of the median nerve in the wrist, is a common disease with a reported uptrend in incidence [5]. Due to compression of the median nerve, CTS patients often experience intractable pain or numbness in the

*Correspondence: aunsittipong@gmail.com

Department of Orthopedics, Faculty of Medicine, Prince of Songkla University, 15 Karnjanavanich Road, Hat Yai, Songkhla 90110, Thailand three radial fingers of the hand and/or develop weakness of intrinsic hand muscles, resulting in functional disability [7, 14]. The disease occurs more commonly in females than males, most commonly in adults aged $30-50$, and is usually bilateral $[1,2]$. There are several known risk factors for CTS, both medical and mechanical [30]. Performing repetitive work using the wrist or handling vibrating instruments for a long time are potential mechanical risk factors. For medical risk factors, hypothyroidism, diabetes mellitus, rheumatoid arthritis, 
and pregnancy also contribute to developing CTS $[1,19]$. In the early stages of CTS, conservative treatment such as oral medications, steroid injections, splinting, therapeutic modalities (ultrasound and iontophoresis), exercise (tendon and nerve gliding), and activity modification [no comma] is the initial approach for alleviating symptoms $[6,31]$. However, some patients do not respond well to conservative treatment and may then progress to carpal tunnel release surgery [3].

Recently some studies have reported a potential linkage between CTS and vitamin D levels, and Vitamin D deficiency is recognized as an independent risk factor for increasing severity of CTS symptoms, particularly tingling pain and nerve function [4, 10,37]. The reason for this connection is that vitamin D, in the form of D2 (ergocalciferol) or D3 (cholecalciferol), has neuroprotection and neurotrophic functions, and also improves nerve myelination which hastens recovery after nerve injuries $[8,9,27]$. Apart from the effect on neurologic function, vitamin D plays a role in suppressing the vascular endothelial growth factor associated with increased inflammatory fibrosis, which may have a role in triggering CTS $[16,17]$. And in relation to pain perception, low vitamin $D$ is related to hypersensitivity of nerve fibers leading to persistent painful neuropathy $[10,20]$.

Eliminating or reducing modifiable risk factors is suggested to reduce the severity of many diseases, including carpel tunnel syndrome. Therefore, correcting low vitamin D levels with vitamin D supplementation is expected to improve treatment outcomes. Previous studies have found that vitamin D levels were correlated with CTS treatment outcomes [29, 32]. However, to date there have been no conclusions or data aggregations concerning the effects of vitamin D supplementation on CTS treatment. The aim of this study was to evaluate earlier studies and summarize whether vitamin D supplementation could improve CTS treatment outcomes.

\section{Materials and methods Search strategy}

We conducted this systematic review according to the guidelines of the Preferred Reporting Items for Systematic Reviews and Meta-Analyses (PRISMA) statement [25]. The search strategy was formulated to focus on the outcomes after vitamin $\mathrm{D}$ supplementation in CTS patients, using the key terms "vitamin D," "D2," "D3," "hypovitaminosis D," or "calciferol" combined with "carpal tunnel syndrome," "CTS," "median nerve entrapment," "median neuritis," "median nerve compression," or "compressive neuropathy." Two investigators (CA and SS) searched for published studies indexed in the PubMed, Cochrane Library, Scopus, and Web of Science databases from January 2000 to March 2021 (Additional file 1). After removing duplicate studies, the eligibility of the remaining studies was independently assessed by two investigators (CA and SS) blinded to the other's decisions on selected studies. Differences were resolved by mutual agreement. If a disagreement persisted, we consulted the third investigator (PS) to arrive at a consensus. Only full articles in English were included.

\section{Eligibility criteria}

All studies which reported an outcome or relation of vitamin D supplementation in CTS patients were reviewed. Original articles written in English were included if they met the following criteria: (1) Cohort (prospective or retrospective) or randomized control trial studies comparing before and after vitamin $\mathrm{D}$ replacement or comparing two groups between CTS patients who received vitamin D supplement and who did not, (2) Patients who had a clinical diagnosis of CTS based on symptoms and specific physical findings, or electrodiagnostic studies, (3) Described the dose and details of vitamin D supplementation, (4) Reported data on the outcome of treatment in terms of clinical conditions or laboratory results, and (5) Follow-up duration not less than three months. No restrictions were set regarding the stage of CTS or route of vitamin D administration. We excluded studies with less than 30 participants. Studies which involved patients who had underlying diseases that needed vitamin D as a treatment were also excluded.

\section{Search results}

From the database search we retrieved a total of 925 studies for evaluation. After removing duplicate results, 522 studies remained and the abstract of each was examined by two investigators (CA and SS). After the abstract reviews 503 studies were excluded as not meeting the inclusion criteria, and another 15 studies were excluded after full-text assessments found no information on the treatment outcomes, leaving four studies for the final analysis (Fig. 1).

\section{Quality assessment}

Two investigators (CA and SS) reviewed and graded each of the four included studies for quality. We applied the Methodological Index for Non-Randomized Studies (MINORS) scoring system to all included studies. Each of the 12 MINORS items is scored 0 to 2, score 0 if not reported, 1 when reported with inadequacy, 2 when reported and adequate. A perfect score was 16 for noncomparative studies and 24 for comparative studies. The quality of each study was categorized according to total scores based on a previous study: $0-5$ points indicated poor quality, $6-10$ points indicated moderate quality, and 11-16 points indicated good quality [11]. 


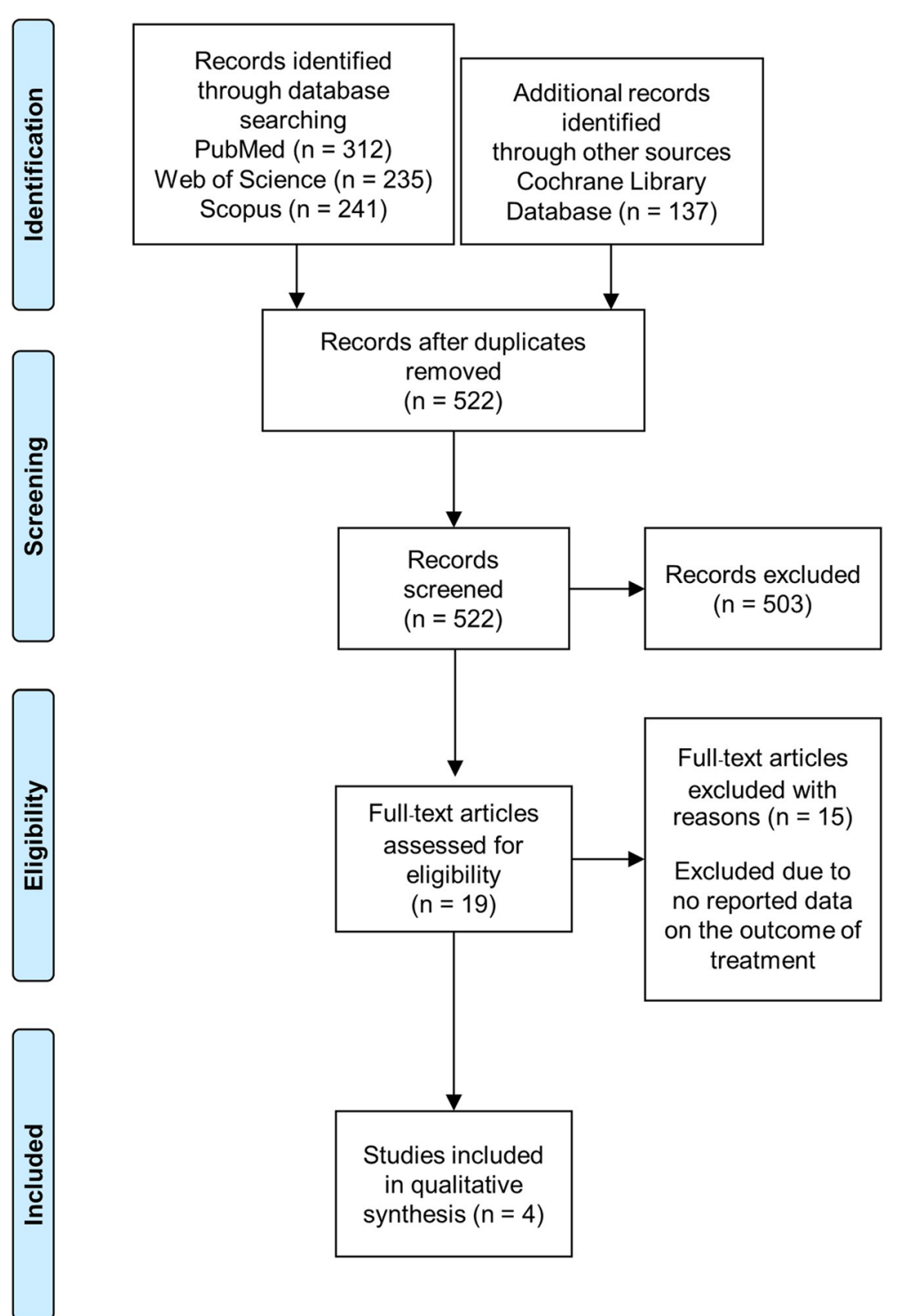

Fig. 1 The flowchart of study selection following the Preferred Reporting Items for Systematic Reviews and Meta-Analyses (PRISMA)

\section{Data collection and extraction}

Duplicate data extraction forms were used for comparison of all outcomes and for achieving completeness with consistency. Any discrepancies were resolved by the senior author (PS). The information in each included study was extracted and recorded. The extracted data about overall study characteristics, patient demographics, including age and gender, and severity of CTS, were evaluated. We also explored the intervention details, including the form and dose of vitamin $\mathrm{D}$ along with the duration of administration. Other related CTS treatments and vitamin D levels between before and after intervention were assessed. We extracted CTS symptoms in pain scores, functional scores, muscle strength, and laboratory findings from electrophysiological results to determine vitamin D supplementation outcomes. Any complications after treatment were also evaluated if available.

\section{Results}

\section{Study characteristics}

Of the four studies, two studies each were retrospective and prospective. All included studies were pre-post studies with no control group or randomization. All studies were recently published, not earlier than 2018, and three were conducted in Asia and one in Europe-Asia (Table 1). For our research question about the outcomes after 
Table 1 Characteristics of included studies

\begin{tabular}{llllllc}
\hline First Author & $\begin{array}{l}\text { Year of } \\
\text { publication }\end{array}$ & Country & Study design & Recruitment Period & $\begin{array}{l}\text { Number of } \\
\text { patients }\end{array}$ & $\begin{array}{c}\text { MINORS scores } \\
\text { Samant et al. [36] }\end{array}$ \\
\hline 2021 & India & Prospective cohort & 2020 & 42 & $11 / 16$ \\
Saçmaci et al. [35] & 2020 & Turkey & Retrospective cohort & $2017-2018$ & 55 & $9 / 16$ \\
Lee et al. [21] & 2019 & Korea & Retrospective cohort & $2014-2016$ & 84 & $7 / 16$ \\
Mohtashamkia et al. [26] & 2018 & Iran & Prospective cohort & 2015 & 50 & $13 / 16$ \\
\hline
\end{tabular}

MINORS Methodological items for non-randomized studies

vitamin D supplementation, all of the included studies were rated as moderate quality with the mean MINORS scores across studies 10 of 16 (range, 7-13). The average MINORS scores in the retrospective studies were 8 of 16 , while the average scores in the prospective studies were 12 of 16. Scores from three studies were deducted from the prospective calculation of the study size and unbiased assessment of the study endpoint (Additional file 2).

\section{Patient demographics and Intervention}

The overall number of patients in the four studies was 231, with 258 wrists included for evaluation. The mean age of patients was between 40 to 60 years, with females predominate even considering one report which did not distinguish between genders [26]. Before vitamin supplementation, three studies reported on the severity of CTS as mild or moderate [26, 35, 36]. Previous and ongoing treatment of CTS were analgesics, anti-inflammatory drugs, splint, physiotherapy, and carpal tunnel release $[21,26,35,36]$. Serum vitamin D levels increased significantly after vitamin supplement in the three studies which reported this $[21,35,36]$, whereas one study did not detail the vitamin D level after supplementation [26]. The doses of oral vitamin $\mathrm{D}$ varied in each study, ranging from 7000 to $60,000 \mathrm{IU} /$ week with a duration of 12 weeks in three studies [26, 35, 36] and 24 weeks in one study [21]. These clinical data and interventions in each included study are presented in Table 2.

\section{Outcomes of vitamin D supplementation: Pain, Function, Nerve conduction}

The various methods for evaluating CTS symptoms, including pain scores, functional scores, muscle strength, and electrophysiological results, are shown in Table 3 . The outcomes before and after the vitamin D supplementation were compared to show the difference in all studies. All studies used combined clinical conditions and nerve conduction velocity to assess the treatment outcomes, except for one study that used only clinical evaluations [36]. The treatment outcomes were evaluated by visual analog scale (VAS) in 124 wrists of 97 patients, functional scores in 176 wrists of 176 patients,

Table 2 Clinical and laboratory data of the patients included in each study

\begin{tabular}{|c|c|c|c|c|c|c|c|c|c|}
\hline \multirow[t]{2}{*}{ First Author } & \multirow{2}{*}{$\begin{array}{l}\text { Number } \\
\text { of wrists }\end{array}$} & \multirow{2}{*}{$\begin{array}{l}\text { Gender } \\
(\mathrm{M} / \mathrm{F})\end{array}$} & \multirow{2}{*}{$\begin{array}{l}\text { Mean age } \\
\text { (years) }\end{array}$} & \multirow[t]{2}{*}{ Severity } & \multicolumn{2}{|c|}{ Serum Vitamin D (ng/ml) } & \multirow{2}{*}{$\begin{array}{l}\text { Form and } \\
\text { Dose }\end{array}$} & \multirow{2}{*}{$\begin{array}{l}\text { Duration } \\
\text { (weeks) }\end{array}$} & \multirow{2}{*}{$\begin{array}{l}\text { Other } \\
\text { Treatments }\end{array}$} \\
\hline & & & & & Before & After & & & \\
\hline $\begin{array}{l}\text { Samant et al. } \\
\text { [36] }\end{array}$ & 42 & $9 / 33$ & $40.88 \pm 7.6$ & $\begin{array}{l}\text { Mild and } \\
\text { Moderate } \\
\text { CTS }\end{array}$ & $13.73 \pm 3.9$ & $46.61 \pm 5.8$ & $\begin{array}{l}\text { Oral Vitamin } \\
\text { D3; dose } \\
60,000 \text { IU/ } \\
\text { week }\end{array}$ & 12 & $\begin{array}{l}\text { Analgesics, } \\
\text { Anti-inflam- } \\
\text { matory drugs, } \\
\text { Splint, Physi- } \\
\text { otherapy }\end{array}$ \\
\hline $\begin{array}{l}\text { Saçmaci et al. } \\
\text { [35] }\end{array}$ & 82 & $0 / 55$ & $\begin{array}{l}48.04 \pm 6.03 \\
\text { in Mild CTS, } \\
45.97 \pm 7.97 \text { in } \\
\text { Moderate CTS }\end{array}$ & $\begin{array}{l}45 \text { Mild CTS } \\
\text { and } 37 \text { Mod- } \\
\text { erate CTS }\end{array}$ & $\begin{array}{l}11.75 \pm 4.53 \\
\text { in Mild CTS, } \\
11.64 \pm 3.55 \text { in } \\
\text { Moderate CTS }\end{array}$ & $\begin{array}{l}39.97 \pm 16.02 \\
\text { in Mild CTS, } \\
32.45 \pm 13.99 \text { in } \\
\text { Moderate CTS }\end{array}$ & $\begin{array}{l}\text { Oral Vitamin } \\
\text { D2 or } \\
\text { D3; dose } \\
6000 \text { IU/day } \\
\text { or 50,000 IU/ } \\
\text { week }\end{array}$ & 12 & Splint \\
\hline Lee et al. [21] & 84 & $0 / 84$ & $56 \pm 9$ & Not reported & $12 \pm 3.6$ & $24.5 \pm 8.5$ & $\begin{array}{l}\text { Oral Vitamin } \\
\text { D; dose } \\
1000 \text { IU/day }\end{array}$ & 24 & $\begin{array}{l}\text { Carpal tunnel } \\
\text { release }\end{array}$ \\
\hline $\begin{array}{l}\text { Mohtasham- } \\
\text { kia et al. [26] }\end{array}$ & 50 & Not reported & $44 \pm 8.5$ & $\begin{array}{l}\text { Mild and } \\
\text { Moderate } \\
\text { CTS }\end{array}$ & $19.1 \pm 6.3$ & Not reported & $\begin{array}{l}\text { Oral Vitamin } \\
\text { D3; dose } \\
50,000 \text { IU/ } \\
\text { week }\end{array}$ & 12 & $\begin{array}{l}\text { Anti-inflam- } \\
\text { matory drugs, } \\
\text { Splint, Physi- } \\
\text { otherapy }\end{array}$ \\
\hline
\end{tabular}




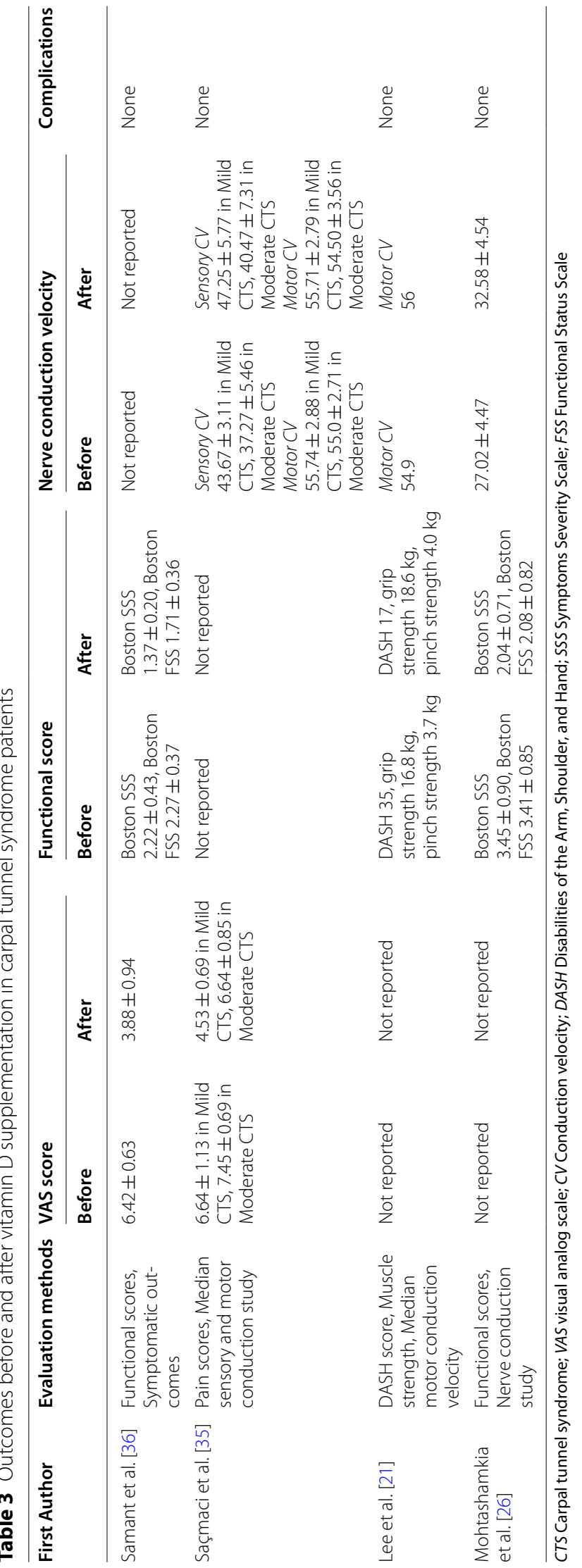


muscle strength in 84 wrists of 84 patients, and nerve conduction velocity in 216 wrists of 189 patients. CTS symptoms as evaluated by VAS scores were decreased significantly in two studies $[35,36]$. Contrary to the pain scores, there were no statistically significant differences in motor power and median motor conduction velocity. However, median sensory conduction velocity was significantly improved [21,35]. The functional outcomes were assessed by the Disabilities of the Arm, Shoulder, and Hand (DASH) scores ranging from 0 (no disability) to 100 (most severe disability), the Boston Symptoms Severity Scale (SSS) ranging from 1 to 5 in 11 items, and the Boston Functional Status Scale (FSS) ranging from 1 to 5 in 8 items. Of the four studies, three of them reported a better functional status after vitamin $\mathrm{D}$ supplementation $[21,26,36]$. There were no reported complications after the intervention in any of the four studies.

\section{Discussion}

Some studies in recent years have reported that hypovitaminosis D is one factor that associated with decreased median nerve function [20,32, 37]. Consequently, some orthopedists have attempted to correct serum vitamin D by prescribing oral vitamin D to improve CTS symptoms. We aggregated recent studies that compared the treatment results between before and after vitamin D supplementation to assess the treatment effect in CTS patients. Although the studies involved only a short period of vitamin $\mathrm{D}$ administration and a wide range of vitamin $\mathrm{D}$ dosages, all studies reported better outcomes after vitamin supplementation in both clinical and laboratory aspects. These studies showed improved symptoms by reduced VAS pain scores and enhanced hand function as assessed by Boston SSS, Boston FSS, and/or DASH scores. We also found favorable results in nerve conduction velocity studies.

\section{Pain scores}

For patients with low serum vitamin D, vitamin supplementation has been found to effectively relieve pain from compressive neuropathy in CTS $[35,36]$. In our systematic review, the pooled VAS scores of 124 wrists reduced from $6.83 \pm 0.95$ to $5.01 \pm 1.44$ out of 10 after vitamin $D$ supplementation. These results can be explained by noting that vitamin D plays a vital role in the pain signaling pathway, with supporting in vivo and in vitro studies [15]. Additionally, vitamin D supplementation has been found to have benefits for acute or chronic pain relief in other orthopedic diseases, including growing pain, low back pain, and osteoarthritis $[13,24,28]$. However, the dosage, duration, and type of vitamin $\mathrm{D}$ for relieving pain are different in each disease.

\section{Functional outcomes}

In the reviewed studies, the specific functional assessment tool for CTS was the Boston Carpal Tunnel Syndrome test, while the non-specific tool was the DASH test. After 12 weeks of vitamin D supplementation, DASH scores were reduced by 18 percent. Consistent with the DASH scores, the pooled Boston SSS scores reduced from $2.89 \pm 0.95$ to $1.73 \pm 0.64$ and the Boston FSS scores reduced from $2.89 \pm 0.88$ to $1.91 \pm 0.68$. Our systematic review highlights that those functional scores of CTS patients were improved after vitamin D supplementation regardless of the specificity of the functional assessment tool. However, grip and pinch strength, indirectly reflecting the muscles innervated by the median nerve, were not changed. These different results may have been due to too short period of vitamin D supplementation to affect the motor functions [34]. There is currently no best standard assessment tool for evaluating functional outcomes. For these reasons, the combination of various reliable tools is recommended to optimize the reliability of evaluating functional status in CTS patients [18]. Although there is a lack of study using multiple functional assessment tools with long-term follow-up, the evidence suggests that vitamin D could enhance CTS patients' functional capacity recovery.

\section{Nerve conduction study}

Conduction abnormalities from nerve compression can be tested by nerve conduction velocity studies, assisting clinicians in either diagnosis or evaluating treatment outcomes [33]. These tests are used to evaluate motor and sensory function. Of the three previous studies observing nerve conduction outcomes, only one study reported results from both types of nerve conduction, with details of latency, amplitude, and conduction velocity [35]. Another of the three studies performed electrophysiological evaluations by motor conduction velocity test alone [21], similar to the previous study [20]. The third reported anonymous nerve conduction velocity results without details [26]. Most of the included patients had mild to moderate CTS severity, which affected the median sensory conduction earlier than motor conduction [1]. In all of these studies, the median nerve conduction velocity results significantly improved after vitamin D supplementation [26, 35]; however, the motor conduction velocity was not noticeably changed, which was in line with non-improved grip and pinch strength [21, 35]. Significant enhancement of motor conduction velocity may occur if the CTS severity exceeds mild-moderate symptoms, or with a more extended vitamin D administration period. This systematic review found that vitamin D supplementation altered only median sensory conduction velocity in mild and moderate CTS severity. 


\section{Complications}

There were no reported complications following vitamin $\mathrm{D}$ administration in this study. Some previous studies reported complications that occurred from iatrogenic accidental overdoses [22]. The clinical presentation of vitamin $\mathrm{D}$ toxicity may range from asymptomatic to lifethreatening features resulting from persistent, prolonged hypercalcemia. Although prescribing the usual therapeutic dose of vitamin D for a long period may lead to hypercalcemia, another study reported that overall adverse events did not noticeably increase [23]. Therefore, oral vitamin $\mathrm{D}$ administration with the appropriate dose is safe for CTS patients.

\section{Limitations}

This review had several limitations. All included studies were cohort studies without a control group, and, according to the methodological index, there were no high-quality studies. Another point about study design is that half of these studies were retrospective, which may have increased the risk of selection and information bias. For these reasons, there could have been a tendency to overestimate the benefits of the intervention. Additionally, only four studies in this systematic review resulted in an overall small number of included patients, even considering the non-restrictive eligibility criteria. And within the small number of patients, they were predominately female, limiting the generalizability of the findings. Regarding the current situation, there are no standardized dosages or forms of vitamin D, whether vitamin D2 or D3, for reducing undesired symptoms in peripheral neuropathy; various vitamin $\mathrm{D}$ doses and forms have been recommended [12,38]. Not only the vitamin D administration but also the concomitant treatments were different in each study, which could have interfered with analysis of the specific effectiveness of vitamin D in CTS. The improved outcomes demonstrated in the studies may have occurred by chance. Finally, these studies had different methods of assessing outcomes. Some used pain scores or functional scores only, whereas some combined these scores with nerve conduction studies. These differences prevented us from directly comparing the outcomes after vitamin D supplementation, which was our primary objective.

More studies are needed to evaluate the influence of vitamin D supplementation in CTS treatment. Future studies should be well-designed, randomized, doubleblind trials, minimizing the risk of bias, comparing outcomes between intervention and control groups, together with controlling potential confounders and assessing outcomes by multiple assessment tools. Also, a longer period of follow-up is required to ensure that the reported improvements are long-term without any adverse events.

\section{Conclusion}

The studies in this systematic review showed that vitamin D supplementation at least 12 weeks could offer favorable outcomes in pain improvement, better functionality, and increased sensory conduction velocity for CTS patients with mild-moderate symptoms. However, there was no standardized dose or duration of vitamin D administration in CTS. Based on current evidence, prescribing the usual appropriate dose of oral vitamin $\mathrm{D}$ as an additional treatment while monitoring serum vitamin $\mathrm{D}$ levels is suggested.

\section{Supplementary Information}

The online version contains supplementary material available at https://doi. org/10.1186/s40634-021-00393-4.

Additional file 1.

Additional file 2 .

\section{Acknowledgements}

The authors sincerely thank Dave Patterson of the International Affairs Department, Prince of Songkla University, for English editing the final draft of this manuscript.

\section{Authors' contributions}

All authors contributed to the study conceptualization and design. Chirathit Anusitviwat and Sitthiphong Suwannaphisit performed study screening, data extraction and assessed study quality. Porames Suwanno resolved any discrepancies in study screening and data extraction. Chirathit Anusitviwat wrote the first draft of the manuscript. Sitthiphong Suwannaphisit edited the manuscript. All authors read and approved the final manuscript.

Funding

No funding was received for this study.

Availability of data and materials

All data generated or analysed during this study are included in this published article and its supplementary information files.

\section{Declarations}

Ethics approval and consent to participate

This systematic review was approved by the Institutional Review Board, Faculty of Medicine, Songklanagarind Hospital, Prince of Songkla University (IRB number 64-159-11-1). Data from individual participants were not obtained.

Consent for publication

Not applicable.

Competing interests

The authors have no relevant financial or non-financial interests to disclose.

Received: 22 April 2021 Accepted: 26 August 2021

Published online: 07 September 2021 


\section{References}

1. Aroori S, Spence RAJ (2008) Carpal tunnel syndrome. Ulster Med J 77:6-17

2. Atroshi I, Gummesson C, Johnsson R, Ornstein E, Ranstam J, Rosén I (1999) Prevalence of carpal tunnel syndrome in a general population. JAMA 282:153-158

3. Baker NA, Livengood HM (2014) Symptom severity and conservative treatment for carpal tunnel syndrome in association with eventual carpal tunnel release. J Hand Surg Am 39:1792-1798

4. Basit A, Basit KA, Fawwad A, Shaheen F, Fatima N, Petropoulos IN, Alam U, Malik RA (2016) Vitamin D for the treatment of painful diabetic neuropathy. BMJ Open Diabetes Res Care 4

5. Burton CL, Chen Y, Chesterton LS, van der Windt DA (2018) Trends in the prevalence, incidence and surgical management of carpal tunnel syndrome between 1993 and 2013: an observational analysis of UK primary care records. BMJ Open Brit Med J 8

6. Calandruccio JH, Thompson NB (2018) Carpal tunnel syndrome: making evidence-based treatment decisions. Orthop Clin North Am 49:223-229

7. Cazares-Manríquez MA, Wilson CC, Vardasca R, García-Alcaraz J, OlguínTiznado JE, López-Barreras JA, García-Rivera BR (2020) A review of carpal tunnel syndrome and its association with age, body mass index, cardiovascular risk factors, hand dominance, and sex.

8. Chabas J-F, Stephan D, Marqueste T, Garcia S, Lavaut M-N, Nguyen C, Legre R, Khrestchatisky M, Decherchi P, Feron F (2013) Cholecalciferol $\left(\right.$ vitamin $\left.D^{3}\right)$ improves myelination and recovery after nerve injury. PLoS One 8

9. DeLuca GC, Kimball SM, Kolasinski J, Ramagopalan SV, Ebers GC (2013) Review: the role of vitamin $\mathrm{D}$ in nervous system health and disease. Neuropathol Appl Neurobiol 39:458-484

10. Demiryurek BE, Gundogdu AA (2017) The effect of vitamin D levels on pain in carpal tunnel syndrome. Orthop Traumatol Surg Res 103:919-922

11. Elkhadem A, Mickan S, Richards D (2014) Adverse events of surgical extrusion in treatment for crown-root and cervical root fractures: a systematic review of case series/reports. Dent Traumatol 30:1-14

12. Faye PA, Poumeaud F, Miressi F, Lia AS, Demiot C, Magy L, Favreau F, Sturtz FG (2019) Focus on 1,25-Dihydroxyvitamin D3 in the peripheral nervous system. Front Neurosci 13:348

13. Ghai B, Bansal D, Kanukula R, Gudala K, Sachdeva N, Dhatt SS, Kumar V (2017) Vitamin D supplementation in patients with chronic low back pain: an open label, single arm clinical trial. Pain Physician 20:E99-E105

14. Ghasemi-Rad M, Nosair E, Vegh A, Mohammadi A, Akkad A, Lesha E, Mohammadi MH, Sayed D, Davarian A, Maleki-Miyandoab T, Hasan A (2014) A handy review of carpal tunnel syndrome: From anatomy to diagnosis and treatment. World J Radiol 6:284-300

15. Habib AM, Nagi K, Thillaiappan NB, Sukumaran V, Akhtar S (2020) Vitamin $\mathrm{D}$ and its potential interplay with pain signaling pathways. Front Immunol 11:820

16. He G, G H, Ja I, Y C, B L, Jr HE (2008) 1,25(OH)2-vitamin D3 inhibits proliferation and decreases production of monocyte chemoattractant protein-1, thrombopoietin, VEGF, and angiogenin by human annulus cells in vitro. Spine (Phila Pa 1976) 33:755-765

17. Hirsch D, Archer FE, Joshi-Kale M, Vetrano AM, Weinberger B (2011) Decreased anti-inflammatory responses to vitamin $D$ in neonatal neutrophils. Mediators Inflamm 2011:598345

18. Jackson D, Zerpa C, Vasiliu D, Sanzo P, Newhouse I (2016) The usefulness of a carpal tunnel compression assessment tool: evidence of reliability and validity in assessing carpal tunnel syndrome. Clinical Practice 5:6-15

19. Karpitskaya Y, Novak CB, Mackinnon SE (2002) Prevalence of smoking, obesity, diabetes mellitus, and thyroid disease in patients with carpal tunnel syndrome. Ann Plast Surg 48:269-273
20. Kuru P, Akyuz G, Yagci I, Giray E (2015) Hypovitaminosis D in widespread pain: its effect on pain perception, quality of life and nerve conduction studies. Rheumatol Int 35:315-322

21. Lee MH, Gong HS, Lee MH, Cho KJ, Kim J, Baek GH (2019) The effect of vitamin D deficiency correction on the outcomes in women after carpal tunnel release. J Hand Surg Am 44:649-654

22. Lim K, Thadhani R, Lim K, Thadhani R (2020) Vitamin D toxicity. Braz J Nephrol Sociedade Brasileira de Nefrologia 42:238-244

23. Malihi Z, Wu Z, Lawes CMM, Scragg R (2019) Adverse events from large dose vitamin D supplementation taken for one year or longer. J Steroid Biochem Mol Biol 188:29-37

24. Manoy P, Yuktanandana P, Tanavalee A, Anomasiri W, Ngarmukos S, Tanpowpong T, Honsawek S (2017) Vitamin D supplementation improves quality of life and physical performance in osteoarthritis patients. Nutrients 9

25. Moher D, Liberati A, Tetzlaff J, Altman DG (2009) Preferred reporting items for systematic reviews and meta-analyses: the PRISMA statement. BMJ Brit Med J 339:b2535

26. Mohtashamkia M, Roshanzamir S, Saghanezhad S (2018) Vitamin D in treatment-resistant CTS patients with hypovitaminosis D. J Clin Diagnostic Res 12:LC23-LC26

27. Montava M, Garcia S, Mancini J, Jammes Y, Courageot J, Lavieille J-P, Feron $F$ (2015) Vitamin D3 potentiates myelination and recovery after facial nerve injury. Eur Arch Otorhinolaryngol 272:2815-2823

28. Morandi G, Maines E, Piona C, Monti E, Sandri M, Gaudino R, Boner A, Antoniazzi F (2015) Significant association among growing pains, vitamin D supplementation, and bone mineral status: results from a pilot cohort study. J Bone Miner Metab 33:201-206

29. Nageeb RS, Shehta N, Nageeb GS, Omran AA (2018) Body mass index and vitamin D level in carpal tunnel syndrome patients. Egypt J Neurol Psychiatr Neurosurg 54:14

30. Newington L, Harris EC, Walker-Bone K (2015) Carpal tunnel syndrome and work. Best Pract Res Clin Rheumatol 29:440-453

31. Osterman AL, Whitman M, Porta LD (2002) Nonoperative carpal tunnel syndrome treatment. Hand Clin 18:279-289

32. Özer G (2018) The impact of serum vitamin D concentration on median nerve conduction. J Clin Exp Invest Modestum 9:63-66

33. Padua L, Coraci D, Erra C, Pazzaglia C, Paolasso I, Loreti C, Caliandro P, Hobson-Webb LD (2016) Carpal tunnel syndrome: clinical features, diagnosis, and management. Lancet Neurol 15:1273-1284

34. Rejnmark $L$ (2011) Effects of vitamin D on muscle function and performance: a review of evidence from randomized controlled trials. Ther Adv Chronic Dis 2:25-37

35. Saçmaci H, Tanik N, Balbaloğlu Ö, Aktürk T, Inan LE (2020) Electrophysiological evaluation of carpal tunnel syndrome female patients after vitamin D replacement. Arq Neuropsiquiatr 78:224-229

36. Samant PD, Sane RM (2021) Evaluation of functional and symptomatic outcomes after vitamin D3 administration in carpal tunnel syndrome with hypovitaminosis D. Hand (N Y) 1558944720988130

37. Tanik N, Balbaloğlu Ö, Ucar M, Sarp U, Atalay T, Çelikbilek A, Göçmen AY, Inan LE (2016) Does vitamin D deficiency trigger carpal tunnel syndrome? J Back Musculoskelet Rehabil 29:835-839

38. Yammine K, Wehbe R, Assi C (2020) A systematic review on the efficacy of vitamin D supplementation on diabetic peripheral neuropathy. Clin Nutr 39:2970-2974

\section{Publisher's Note}

Springer Nature remains neutral with regard to jurisdictional claims in published maps and institutional affiliations. 